Pacific

Journal of

Mathematics

CONSTRUCTION DE LA TOUR DES 2-CORPS DE CLASSES DE HILBERT DE CERTAINS CORPS BIQUADRATIQUES

AbDelmaleK Azizi 


\title{
CONSTRUCTION DE LA TOUR DES 2-CORPS DE CLASSES DE HILBERT DE CERTAINS CORPS BIQUADRATIQUES
}

\author{
ABDELMALEK AZIZI
}

Let $p$ and $q$ be prime numbers such that $p \equiv 1 \bmod 8, q \equiv$ $-1 \bmod 4$ and $\left(\frac{p}{q}\right)=-1, d=p q, \mathrm{k}=\mathrm{Q}(\sqrt{d}, i), \mathrm{k}_{2}^{(1)}$ be the 2-Hilbert class field of $\mathrm{k}, \mathrm{k}_{2}^{(2)}$ be the 2-Hilbert class field of $\mathrm{k}_{2}^{(1)}$ and $G_{2}$ be the Galois group of $\mathrm{k}_{2}^{(2)} / \mathrm{k}$. The 2-part $C_{\mathrm{k}, 2}$ of the class group of $\mathrm{k}$ is of type $(2,2)$, so $\mathrm{k}_{2}^{(1)}$ contains three extensions $\mathrm{K}_{i} / \mathrm{k}, i=1,2,3$. Our goal is to determine the group $C_{\mathrm{k}, 2}$, to study the problem of capitulation of the 2classes of $\mathrm{k}$ in $\mathrm{K}_{i}, i=1,2,3$ and to construct the 2-class field tower of $\mathrm{k}$.

Résumé.

Soient $p$ et $q$ deux nombres premiers tels que $p \equiv 1 \bmod$ $8, q \equiv-1 \bmod 4$ et $\left(\frac{p}{q}\right)=-1, d=p q, i=\sqrt{-1}, \mathrm{k}=$ $\mathrm{Q}(\sqrt{d}, i), \mathrm{k}_{2}^{(1)}$ le 2-corps de classes de Hilbert de $\mathrm{k}, \mathrm{k}_{2}^{(2)}$ le 2-corps de classes de Hilbert de $\mathrm{k}_{2}^{(1)}$ et $G_{2}$ le groupe de Galois de $\mathrm{k}_{2}^{(2)} / \mathrm{k}$. La 2-partie $C_{\mathrm{k}, 2}$, du groupe de classes de $\mathrm{k}$ est de type $(2,2)$, par suite $\mathrm{k}_{2}^{(1)}$ contient trois extensions $\mathrm{K}_{i} / \mathrm{k}, i=1,2,3$. On s'intéresse à déterminer le groupe $C_{\mathrm{k}, 2}$, à etudier la capitulation des 2-classes de $\mathrm{k}$ dans $\mathrm{K}_{i}, i=1,2,3$ et à la construction de la tour du 2-corps de classes de Hilbert de $k$.

\section{Introduction.}

Soient $\mathbf{k}$ un corps de nombres de degré fini sur $\mathbf{Q}, \mathbf{F}$ une extension non ramifiée de $\mathbf{k}$ et $p$ un nombre premier. L'extension $\mathbf{k}^{(1)}$ de $\mathbf{k}$, abélienne maximale et non-ramifiée pour tous les idéaux premiers finis et infinis, est dite corps de classes de Hilbert de $\mathbf{k}$. De même l'extension $\mathbf{k}_{p}^{(1)}$ de $\mathbf{k}$ dont le degré est une puissance de $p$, abélienne maximale et non-ramifiée pour tous les idéaux premiers finis et infinis est dite $p$-corps de classes de Hilbert de $\mathbf{k}$.

La recherche des idéaux de $k$ qui capitulent dans $\mathbf{F}$ (deviennent principaux dans $\mathbf{F}$ ), a été l'objet d'étude d'un grand nombre de mathématiciens. En effet, Kronecker était parmi les premiers à avoir abordé des problèmes de capitulation dans le cas des corps quadratiques imaginaires. Dans le cas 
où $\mathbf{F}$ est égal au corps de classes de Hilbert $\mathbf{k}^{(1)}$ de $\mathbf{k}$, D. Hilbert avait conjecturé que toutes les classes de $\mathbf{k}$ capitulent dans $\mathbf{k}^{(1)}$ (théorème de l'idéal principal). La preuve de ce dernier théorème a été réduite par E. Artin à un problème de la théorie des groupes, et c'est $\mathrm{Ph}$. Furtwängler qui l'avait achevée.

Le cas où $\mathbf{F} / \mathbf{k}$ est une extension cyclique et $[\mathbf{F}: \mathbf{k}]=p$, un nombre premier, a été traité par Hilbert. Sa réponse est le sujet du Théorème 94 qui affirme qu'il y a au moins une classe non-triviale dans $\mathbf{k}$ qui capitule dans $\mathbf{F}$. De plus, Hilbert avait trouvé le résultat suivant:

Soient $\sigma$ un générateur du groupe de Galois de $\mathbf{F} / \mathbf{k}, N$ la norme de $\mathbf{F} / \mathbf{k}$, $\mathbf{U}_{0}$ le groupe des unités de $\mathbf{k}$, $\mathbf{U}$ le groupe des unités de $\mathbf{F}$ et $\mathbf{U}^{*}$ le sousgroupe des unités de $\mathbf{U}$ dont la norme, relative à l'extension $\mathbf{F} / \mathbf{k}$, est égale à 1. Alors le groupe des classes de $\mathbf{k}$ qui capitulent dans $\mathbf{F}$ est isomorphe au groupe quotient $\mathbf{U}^{*} / \mathbf{U}^{1-\sigma}=\mathbf{H}^{1}(\mathbf{U})$, le groupe cohomologique de $\mathbf{U}$ de dimension 1.

A l'aide de ce théorème et de plusieurs résultats sur les groupes cohomologiques des unités, on montre le théorème suivant:

Théorème 1. Soit $\mathbf{F} / \mathbf{k}$ une extension cyclique de degré un nombre premier, alors le nombre des classes qui capitulent dans $\mathbf{F} / \mathbf{k}$ est égal à

$$
[\mathbf{F}: \mathbf{k}]\left[\mathbf{U}_{0}: N(\mathbf{U})\right] \text {. }
$$

On trouve une preuve de ce théorème dans un papier de Heider et Schmithals $[\mathbf{1 1}]$.

Plusieurs résultats ont été établis; en particulier on a:

Soit $\mathbf{k}$ tel que $C_{\mathbf{k}, 2}$, la 2-partie du groupe des classes $C_{\mathbf{k}}$ de $\mathbf{k}$, est isomorphe à $\mathbf{Z} / 2 \mathbf{Z} \times \mathbf{Z} / 2 \mathbf{Z}, \mathbf{k}_{2}^{(2)}$ le 2-corps de classes de Hilbert de $\mathbf{k}_{2}^{(1)}$ et $G_{2}$ le groupe de Galois de $\mathbf{k}_{2}^{(2)} / \mathbf{k}$. On sait par la théorie des corps de classes que $\operatorname{Gal}\left(\mathbf{k}_{2}^{(1)} / \mathbf{k}\right) \simeq C_{\mathbf{k}, 2}$, par suite $\operatorname{Gal}\left(\mathbf{k}_{2}^{(1)} / \mathbf{k}\right) \simeq \mathbf{Z} / 2 \mathbf{Z} \times \mathbf{Z} / 2 \mathbf{Z}$. Alors $\mathbf{k}_{2}^{(1)}$ contient trois extensions quadratiques de $\mathbf{k}$ dénotées par $\mathbf{K}_{1}, \mathbf{K}_{2}$ et $\mathbf{K}_{3}$.

D'après Kisilevsky [12] on a:

Théorème 2. Soient $\mathbf{k}$ tel que $C_{\mathbf{k}, 2} \simeq \mathbf{Z} / 2 \mathbf{Z} \times \mathbf{Z} / 2 \mathbf{Z}$ et $G_{2}$ le groupe de Galois de $\mathbf{k}_{2}^{(2)} / \mathbf{k}$; alors on a trois types de capitulation:

Type 1. Les quatre classes de $C_{\mathbf{k}, 2}$ capitulent dans chacune des extensions $\mathbf{K}_{i} / \mathbf{k}, i=1,2,3$. Ceci est possible si et seulement si $\mathbf{k}_{2}^{(2)}=\mathbf{k}_{2}^{(1)}$.

Type 2. Les quatre classes de $C_{\mathbf{k}, 2}$ capitulent toutes seulement dans une extension parmi les trois extensions $\mathbf{K}_{i} / \mathbf{k}, i=1,2$, 3. Dans ce cas le groupe $G_{2}$ est diédral.

Type 3. Seulement deux classes capitulent dans chacune des extensions $\mathbf{K}_{i} / \mathbf{k}, i=1,2,3$. Dans ce cas le groupe $G_{2}$ est semidiédral ou quaternionique. 
Soit $G_{2}^{\prime}$ le groupe dérivé de $G_{2}$, si $C_{\mathbf{k}, 2} \simeq \mathbf{Z} / 2 \mathbf{Z} \times \mathbf{Z} / 2 \mathbf{Z}$, alors on a de même $G_{2} / G_{2}^{\prime} \simeq \mathbf{Z} / 2 \mathbf{Z} \times \mathbf{Z} / 2 \mathbf{Z}$; ce qui implique que $G_{2}^{\prime}$ est cyclique. Comme $G_{2}^{\prime} \simeq \operatorname{Gal}\left(\mathbf{k}_{2}^{(2)} / \mathbf{k}_{2}^{1}\right)$ est cyclique, alors la tour des 2-corps de classes de Hilbert de $\mathbf{k}$ s'arrête en $\mathbf{k}_{2}^{(2)}$. Donc pour construire la tour des 2-corps de classes de Hilbert, il suffit de construire $\mathbf{k}_{2}^{(1)}$ et $\mathbf{k}_{2}^{(2)}$. On trouve plusieurs travaux sur la construction des 2-corps de classes de Hilbert; en particulier on trouve les travaux de $\mathrm{H}$. Cohn dans [9] et dans [10], qui a construit le 2-corps de classes de Hilbert de $\mathbf{Q}(\sqrt{-p})$ où $p$ est un premier tel que $p \equiv 1 \bmod 4$.

Dans toute la suite on désigne par $p$ et $q$ deux nombres premiers tels que $p \equiv 1 \bmod 8, q \equiv-1 \bmod 4$ et $\left(\frac{p}{q}\right)=-1, d=p q, \mathbf{k}=\mathbf{Q}(\sqrt{d}, i), \mathbf{k}_{2}^{(1)}$ le 2-corps de classes de Hilbert de $\mathbf{k}, \mathbf{k}_{2}^{(2)}$ le 2-corps de classes de Hilbert de $\mathbf{k}_{2}^{(1)}$ et $G_{2}$ le groupe de Galois de $\mathbf{k}_{2}^{(2)} / \mathbf{k}$. D'après Azizi [2], on a $C_{\mathbf{k}, 2} \simeq$ $\mathbf{Z} / 2 \mathbf{Z} \times \mathbf{Z} / 2 \mathbf{Z}$. Donc $\mathbf{k}_{2}^{(1)}$ contient trois extensions quadratiques de $\mathbf{k}, \mathbf{K}_{1}$, $\mathbf{K}_{2}$ et $\mathbf{K}_{3}$. Notre but est de déterminer $C_{\mathbf{k}, 2}$, d'étudier la capitulation dans les trois extensions $\mathbf{K}_{i} / \mathbf{k}, i=1,2,3$ et de construire la tour des 2-corps de classes de Hilbert de $\mathbf{k}$. En particulier on a le résultat principal suivant:

Théorème 3. Soient $\epsilon$ l'unité fondamentale de $\mathbf{Q}(\sqrt{p}), \mathbf{L}=\mathbf{Q}(\sqrt{-p})$ et $\mathbf{L}_{2}^{(1)}$ le 2-corps de classes de Hilbert de $\mathbf{L}$. Alors il existe deux entiers $a, b \in$ $\mathbf{N}$ tels que $p=a^{2}+16 b^{2}$. Soient $\pi_{1}=a+4 b i, \pi_{2}=a-4 b i, \mathcal{H}_{1}$ et $\mathcal{H}_{2}$ les idéaux premiers au-dessus de $\pi_{1}$ et $\pi_{2}$ dans $\mathbf{k}$. Alors la 2-partie du groupe de classes de $\mathbf{k}$ est engendré par les classes de $\mathcal{H}_{1}$ et $\mathcal{H}_{2}$ et les trois extensions quadratiques non-ramifiées sur $\mathbf{k}$ sont: $\mathbf{k}^{(*)}=\mathbf{k}(\sqrt{p}), \mathbf{K}_{1}=\mathbf{k}\left(\sqrt{\pi_{1}}\right)$ et $\mathbf{K}_{2}=$ $\mathbf{k}\left(\sqrt{\pi_{2}}\right)$. De plus si $\mathbf{k}_{2}^{(2)} \neq \mathbf{k}_{2}^{(1)}$, alors seules la classe de $\mathcal{H}_{1}$ et son carré capitulent dans $\mathbf{K}_{1}$ et il en est de même pour $\mathbf{K}_{2}$, c'est-à-dire seules la classe de $\mathcal{H}_{2}$ et son carré capitulent dans $\mathbf{K}_{2}$. De plus on a $\mathbf{k}_{2}^{(1)}=\mathbf{k}(\sqrt{p})(\sqrt{\epsilon})$ et $\mathbf{k}_{2}^{(2)}=\mathbf{k}^{(*)} \mathbf{L}_{2}^{(1)}$.

\section{Capitulation dans le corps de genres de $\mathrm{k}$.}

Soient $p$ et $q$ deux nombres premiers, $d=p q$ et $\mathbf{k}=\mathbf{Q}(\sqrt{d}, i)$. Dans toute la suite on supposera que $p \equiv 1 \bmod 8, q \equiv-1 \bmod 4$ et $\left(\frac{p}{q}\right)=-1$. Soient $\mathbf{k}_{2}^{(1)}$ le 2-corps de classes de Hilbert de $\mathbf{k}$ et $\mathbf{k}^{(*)}$ le corps des genres de $\mathbf{k}$ (c'est l'extension maximale non-ramifiée pour tous les idéaux premiers, finis et infinis, et qui est abélienne sur $\mathbf{Q}$ ). 


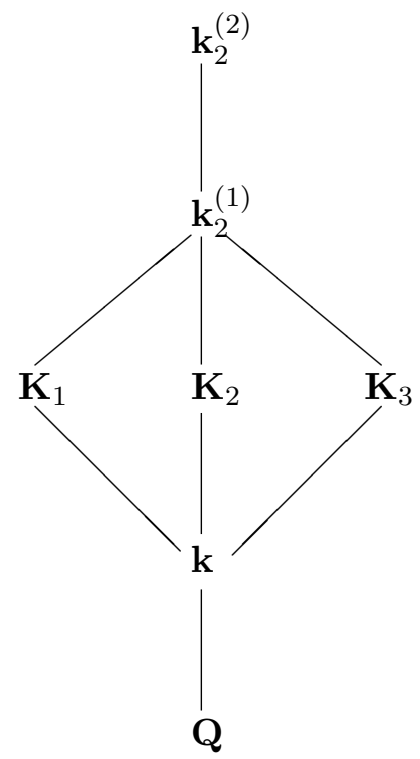

\section{Diagramme 1.}

Le corps des genres de $\mathbf{k}$ est $\mathbf{k}^{(*)}=\mathbf{Q}(\sqrt{p}, \sqrt{q}, i)$. D'après Azizi [6], on a les deux résultats suivants:

Théorème 4. Soient $\mathbf{k}=\mathbf{Q}(\sqrt{p q}, i)$ avec $p$ et $q$ deux nombres premiers tels que $q \equiv-1 \bmod 4, p \equiv 1 \bmod 8,\left(\frac{p}{q}\right)=-1, \mathbf{k}^{(*)}=\mathbf{Q}(\sqrt{p}, \sqrt{q}, i)$ le corps des genres de $\mathbf{k}, \mathbf{k}_{2}^{(1)}$ le 2-corps de classes de Hilbert de $\mathbf{k}, \mathbf{k}_{2}^{(2)}$ le 2-corps de classes de Hilbert de $\mathbf{k}_{2}^{(1)}$ et $\mathbf{C}_{\mathbf{k}, 2}$ la 2-partie du groupe des classes au sens large de $\mathbf{k}$. Alors on a:

1. toutes les classes de $\mathbf{C}_{\mathbf{k}, 2}$ capitulent dans $\mathbf{k}^{(*)}$.

2. $\mathbf{k}_{2}^{(2)} \neq \mathbf{k}_{2}^{(1)} \Leftrightarrow 4 \mid h\left(\mathbf{k}^{(*)}\right) \Leftrightarrow p=x^{2}+32 y^{2}$.

Corollaire 5. Soit $\mathbf{k}=\mathbf{Q}(\sqrt{p q}, i)$ avec $p$ et $q$ deux nombres premiers tels que $q \equiv-1 \bmod 4, p \equiv 1 \bmod 8,\left(\frac{p}{q}\right)=-1$. Soit $G_{2}$ le groupe de Galois de $\mathbf{k}_{2}^{(2)} / \mathbf{k}$. Alors le groupe $G_{2}$ est de type $(2,2)$ ou bien diédral d'ordre $2^{m}$. De plus, $G_{2}$ est diédral si et seulement si $p=x^{2}+32 y^{2}$ avec $x$ et $y$ deux entiers naturels.

D'après le Théorème 4 , toutes les classes de $\mathbf{C}_{\mathbf{k}, 2}$ capitulent dans $\mathbf{k}^{(*)}$ et d'après le Théorème 2 on a les deux possibilités suivantes:

i) si $\mathbf{k}_{2}^{(2)}=\mathbf{k}_{2}^{(1)}$, alors toutes les classes de $\mathbf{C}_{\mathbf{k}, 2}$ capitulent dans $\mathbf{K}_{1}$ et dans $\mathbf{K}_{2}$.

ii) si $\mathbf{k}_{2}^{(2)} \neq \mathbf{k}_{2}^{(1)}$, alors seulement une classe non-triviale de $\mathbf{C}_{\mathbf{k}, 2}$ capitule dans $\mathbf{K}_{1}$ et il en est de même pour $\mathbf{K}_{2}$. 
Dans toute la suite on va déterminer le groupe $\mathbf{C}_{\mathbf{k}, 2}$ et mettre le point sur les classes qui capitulent dans $\mathbf{K}_{1}$ et celles qui capitulent dans $\mathbf{K}_{2}$.

\section{Capitulation de type classe et construction de la tour des 2-corps de classes de Hilbert.}

Soient $d=p q$ avec $p$ et $q$ deux premiers tels que $p \equiv 1 \bmod 8, q \equiv-1 \bmod 4$ et $\left(\frac{p}{q}\right)=-1, \mathbf{k}=\mathbf{Q}(\sqrt{d}, i), \mathbf{C}_{\mathbf{k}, 2}$ le 2 -groupe des classes de $\mathbf{k}, \mathbf{E}_{\mathbf{k}}$ le groupe des unités de $\mathbf{k}, \mathbf{k}_{2}^{(1)}$ le 2-corps de classes de Hilbert de $\mathbf{k}, \mathbf{k}^{(*)}$ le corps des genres de $\mathbf{k}, H$ le groupe de Galois de $\mathbf{k}_{2}^{(1)} / \mathbf{k}$ et $\hat{H}$ le groupe des caractères de $H$. Soit $\mathbf{L}$ une extension quadratique de $\mathbf{k}$. Alors il existe un nombre $\alpha$ de $\mathbf{k}$ tel que $\mathbf{L}=\mathbf{k}(\sqrt{\alpha})$. Si $\mathbf{L} / \mathbf{k}$ est non-ramifié aux idéaux premiers finis, alors on peut choisir $\alpha$ premier avec 2 tel que:

i) il existe un idéal $\mathcal{H}$ tel que $\mathcal{H}^{2}=(\alpha)$;

ii) il existe $x \in \mathbf{k}$ tel que $\alpha \equiv x^{2} \bmod 4$.

Cette dernière condition est satisfaite si et seulement si $\alpha=x^{2}+4 \frac{r}{s}$, où $\mathrm{r}$ et $\mathrm{s}$ sont des entiers de $\mathbf{k}$ et $\mathbf{s}$ est premier avec 2 .

Soient $\overline{R_{\mathbf{k}}}$ l'ensemble de tous les nombres $\alpha$ de $\mathbf{k}$ vérifiant les deux conditions précédentes, $R_{\mathbf{k}}=\overline{R_{\mathbf{k}}} / \overline{R_{\mathbf{k}}} \cap\left(\mathbf{k}^{*}\right)^{2}$ et $U_{\mathbf{k}}$ l'ensemble des unités de $\mathbf{k}$ appartenant à $\overline{R_{\mathrm{k}}}$.

Définition 6. On dit que $\mathbf{k}$ est de type classe si et seulement si $U_{\mathbf{k}}=\mathbf{E}_{\mathbf{k}}^{2}$. Dans le cas contraire on dit que $\mathbf{k}$ est de type unité.

On définit un homomorphisme $\varphi$ de $R_{\mathbf{k}}$ dans $\mathbf{C}_{\mathbf{k}, 2}$ de la façon suivante: À une classe de $R_{\mathbf{k}}$ d'un nombre $\alpha$ on fait correspondre la classe de l'idéal $\mathcal{H}$ tel que $\mathcal{H}^{2}=(\alpha)$. Alors on a $\operatorname{Ker}(\varphi)=U_{\mathbf{k}} / \mathbf{E}_{\mathbf{k}}^{2}$ et $\mathbf{k}$ est de type classe si et seulement si $\operatorname{Im}(\varphi)$ est égal au sous-groupe du groupe des classes au sens restreint, engendré par les éléments d'ordre deux.

Proposition 7. Soient $\mathbf{k}^{(*)}=\mathbf{k}(\sqrt{p})$ le corps des genres de $\mathbf{k}$ et $\mathcal{H}_{0}$ l'idéal de $\mathbf{k}$ tel que $\mathcal{H}_{0}^{2}=(p)$. Si $\mathbf{k}$ est de type classe, alors la classe de l'idéal $\mathcal{H}_{0}$ est d'ordre 2 . Si $\left(\pi_{1}\right)$ et $\left(\pi_{2}\right)$ sont les deux idéaux premiers au-dessus de $p$ dans $\mathbf{Q}(i)$ et $\mathcal{H}_{1}$ (resp. $\mathcal{H}_{2}$ ) est un idéal premier au-dessus de $\pi_{1}$ (resp. $\left.\pi_{2}\right)$ dans $\mathbf{k} / \mathbf{Q}(i)$, alors les classes de $\mathcal{H}_{1}$ et de $\mathcal{H}_{2}$ engendrent $\mathbf{C}_{\mathbf{k}, 2}$ et on a $\mathcal{H}_{1} \mathcal{H}_{2}=\mathcal{H}_{0}$.

Preuve. Comme $p \equiv 1 \bmod 4$, alors il existe deux nombres $\pi_{1}$ et $\pi_{2}$ de $\mathbf{Q}(i)$ tels que $\pi_{1} \pi_{2}=p$. De plus, puisque $p$ est ramifié dans $\mathbf{k} / \mathbf{Q}(i)$, alors il existe deux idéaux de $\mathbf{k}, \mathcal{H}_{1}$ et $\mathcal{H}_{2}$ tels que $\mathcal{H}_{1}^{2}=\left(\pi_{1}\right), \mathcal{H}_{2}^{2}=\left(\pi_{2}\right)$ et $\left(\mathcal{H}_{1} \mathcal{H}_{2}\right)^{2}=(p)=\mathcal{H}_{0}^{2}$. D'où $\mathcal{H}_{1} \mathcal{H}_{2}=\mathcal{H}_{0}$. Si $\mathbf{k}$ est de type classe, la classe de l'idéal $\mathcal{H}_{0}$ dans $\mathbf{C}_{\mathbf{k}, 2}$ est d'ordre 2 , car sinon, il existe un $\beta \in \mathbf{k}$ tel que $\mathcal{H}_{0}=(\beta)$ et $\left(\beta^{2}\right)=(p)$. Il s'ensuit que $p=\beta^{2} \epsilon$ pour une certaine unité $\epsilon$ de $\mathbf{k}$. Comme $\mathbf{k}(\sqrt{p})=\mathbf{k}(\sqrt{\epsilon})$ est non-ramifié pour tous les idéaux premiers, alors $\epsilon \in U_{\mathbf{k}}$ et $\epsilon \notin \mathbf{E}_{\mathbf{k}}^{2}$, ce qui est contraire au fait que $\mathbf{k}$ est de type classe. 
Il vient que les classes de $\mathcal{H}_{1}$ et $\mathcal{H}_{2}$ sont d'ordre 2 et comme leur produit est d'ordre 2 , alors leurs classes engendrent $\mathbf{C}_{\mathbf{k}, 2}$.

Proposition 8. Soient a un entier composé, impair et sans facteurs carrés, $\mathbf{k}=\mathbf{Q}(\sqrt{a}, i), p$ un nombre premier et $\mathcal{H}$ un idéal de $\mathbf{k}$ tel que $\mathcal{H}^{2}=(p)$. Alors on a:

i) Si l'unité fondamentale de $\mathbf{Q}(\sqrt{a})$ est de norme -1 , alors $\mathcal{H}$ est d'ordre 2 dans $\mathbf{C}_{\mathbf{k}, 2}$.

ii) Si l'unité fondamentale de $\mathbf{Q}(\sqrt{a}), \epsilon_{0}=s+t \sqrt{a}$, est de norme 1 on a:

a) $S i\left\{\epsilon_{0}\right\}$ est un $S F U$ de $\mathbf{k}$, alors $\mathcal{H}$ est principal si et seulement si $2 p(s \pm 1)$ ou $p(s \pm 1)$ est un carré dans $\mathbf{N}$.

b) Sinon, l'idéal $\mathcal{H}$ est d'ordre 2 dans $\mathbf{C}_{\mathbf{k}, 2}$.

Preuve. Soient $p$ un nombre premier et $\mathcal{H}$ un idéal de $\mathbf{k}$ tel que $\mathcal{H}^{2}=(p)$. On suppose que $\mathcal{H}$ est principal. Il existe $\beta \in \mathbf{k}$ et $\epsilon$ une unité de $\mathbf{k}$ tels que $\beta^{2}=p \epsilon$. Nous déterminons les conditions pour que $p \epsilon$ soit un carré dans $\mathbf{k}$. Soit $\epsilon_{0}=s+t \sqrt{a}$ l'unité fondamentale de $\mathbf{Q}(\sqrt{a})$. Alors un SFU de $\mathbf{k}$ est $\left\{\epsilon_{0}\right\}$ ou $\left\{\sqrt{i \epsilon_{0}}\right\}$.

Cas où $\left\{\epsilon_{0}\right\}$ est un SFU de $\mathbf{k}$ :

On se ramène aux cas où $\epsilon$ est égal à $i, \epsilon_{0}$ ou à $i \epsilon_{0}$.

$\star$ Si $\epsilon=\epsilon_{0}$, alors il existe $\left(\beta_{1}, \beta_{2}\right) \in \mathbf{Q}(\sqrt{a})^{2}$ tel que $p \epsilon_{0}=\beta^{2}=\left(\beta_{1}+\right.$ $\left.\beta_{2} i\right)^{2}=\beta_{1}^{2}-\beta_{2}^{2}+2 \beta_{1} \beta_{2} i$. D'où

$$
\left\{\begin{array} { l } 
{ \beta _ { 1 } \beta _ { 2 } = 0 } \\
{ \beta _ { 1 } ^ { 2 } - \beta _ { 2 } ^ { 2 } = p \epsilon _ { 0 } }
\end{array} , \text { ce qui est équivalent à } \left\{\begin{array}{l}
\beta_{2}=0 \\
\beta_{1}^{2}=p \epsilon_{0} .
\end{array}\right.\right.
$$

On pose $\beta_{1}=x+y \sqrt{a}$. Alors on a:

$\left\{\begin{array}{l}x^{2}+a y^{2}=p s \\ 2 x y=p t\end{array}\right.$, ce qui est équivalent à $\left\{\begin{array}{l}4 x^{4}-4 p s x^{2}+a p^{2} t^{2}=0 \\ y=\frac{p t}{2 x} \\ \Delta=16 p^{2}\left(s^{2}-a t^{2}\right) .\end{array}\right.$

Le nombre $\Delta$ est le discriminant de l'équation du deuxième degré $4 x^{4}-$ $4 p s x^{2}+a p^{2} t^{2}=0$ pour l'indéterminée $x^{2}$. On désigne cette dernière équation par (1).

- Si $\epsilon_{0}$ est de norme -1 alors $\Delta$ est négatif. Donc il n'y a pas de solutions pour l'équation (1).

- Si $\epsilon_{0}$ est de norme 1 , alors $x^{2}=\frac{2 p s \pm 2 p}{4}$. Par suite, il y a une solution pour l'équation (1) si et seulement si $2 p(s \pm 1)$ est un carré dans $\mathbf{N}$.

* Soit $\epsilon=i \epsilon_{0}$. De la même façon, on se ramène à $2 p \epsilon_{0}=\gamma^{2}$ où $\gamma \epsilon$ $\mathbf{Q}(\sqrt{a})$ et on trouve des résultats similaires:

- Si $\epsilon_{0}$ est de norme -1, il n'y a pas de solutions.

- Sinon, il y a une solution si et seulement si $p(s \pm 1)$ est un carré dans $\mathbf{N}$. 
$\star$ Soit $\epsilon=i$. On a $p i=\beta^{2} \Leftrightarrow p=2 \beta_{1}^{2}$ où $\beta_{1}$ est la partie réelle de $\beta$. Or ceci implique que $\sqrt{2 p} \in \mathbf{Q}(\sqrt{a})$, ce qui n'est pas notre cas.

Cas où $\left\{\sqrt{i \epsilon_{0}}\right\}$ est un SFU de $\mathbf{k}$ :

Soit l'équation $p \epsilon=\beta^{2}$. Alors on se ramène aux cas: $p^{2} \epsilon^{2}=\beta^{4}$ et $\epsilon^{2}= \pm i \epsilon_{0}, \epsilon=i, \epsilon=i \epsilon_{0}$ ou $\epsilon=\epsilon_{0}$.

- Si $\epsilon^{2}= \pm i \epsilon_{0}$, on a $\beta=\beta_{1}+i \beta_{2}$ et $\pm i p^{2} \epsilon_{0}=\left(\beta_{1}^{2}-\beta_{2}^{2}\right)^{2}-4\left(\beta_{1} \beta_{2}\right)^{2}+$ $4 \beta_{1} \beta_{2}\left(\beta_{1}^{2}-\beta_{2}^{2}\right) i$. D'où $\left(\beta_{1}^{2}-\beta_{2}^{2}\right)^{2}-4\left(\beta_{1} \beta_{2}\right)^{2}=\left(\beta_{1}^{2}-\beta_{2}^{2}-2 \beta_{1} \beta_{2}\right)\left(\beta_{1}^{2}-\right.$ $\left.\beta_{2}^{2}+2 \beta_{1} \beta_{2}\right)=0$ et donc on a $\left(\beta_{1}-\beta_{2}\right)^{2}=2 \beta_{2}^{2}$ ou $\left(\beta_{1}+\beta_{2}\right)^{2}=2 \beta_{2}^{2}$, ce qui entraîne que $\sqrt{2} \in \mathbf{Q}(\sqrt{a})$. Mais ceci n'est pas notre cas.

- Si $\epsilon=i$, avec le même raisonnement que précédemment on trouve que le nombre $p i$ n'est pas un carré dans $\mathbf{k}$.

- Si $\epsilon=i \epsilon_{0}$, alors $p i \epsilon_{0}=\beta^{2}$ implique que $p$ est un carré dans $\mathbf{Q}(\sqrt{a})$. Ceci n'est pas notre cas.

- Si $\epsilon=\epsilon_{0}$, on se ramène au cas $\epsilon=i$ (car $i \epsilon_{0}$ est un carré dans $\mathbf{k}$ ).

Proposition 9. Soit $\mathbf{k}=\mathbf{Q}(\sqrt{d}, i)$ où $d=p q$ avec $p \equiv 1 \bmod 8, q \equiv$ $-1 \bmod 4,\left(\frac{p}{q}\right)=-1$. Alors $\mathbf{k}$ est de type classe.

Preuve. D'après Azizi $[\mathbf{6}],\left\{\sqrt{i \epsilon_{0}}\right\}$ est un SFU de $\mathbf{k}$, donc d'après la proposition précédente, l'idéal $\mathcal{H}$ tel que $\mathcal{H}^{2}=(p)$ est d'ordre 2 dans $\mathbf{C}_{\mathbf{k}, 2}$. Par suite $\mathbf{k}^{(*)}$ n'est pas de la forme $\mathbf{k}(\sqrt{\epsilon})$ où $\epsilon$ est une unité de $\mathbf{k}$. On suppose que $\mathbf{k}$ est de type unité. Il existe une unité $\epsilon$ de $\mathbf{k}$ telle que $\mathbf{k}(\sqrt{\epsilon})$ soit non-ramifié sur $\mathbf{k}$. En particulier, il existe $x$ dans $\mathbf{k}, \mathrm{r}$ et $\mathrm{s}$ deux entiers de $\mathbf{k}$ tels que $\mathrm{s}$ est premier avec 2 et $\epsilon=x^{2}+4 \frac{r}{s}$. On désigne par $\epsilon_{1}=\sqrt{i \epsilon_{0}}$ et $\sigma$ l'automorphisme de $\mathbf{k}$ défini par $\sigma(i)=i$ et $\sigma(\sqrt{d})=-\sqrt{d}$. L'unité $\epsilon$ ne peut pas être égale à $i$, car sinon, $\mathbf{k}(\sqrt{2})$ sera non-ramifié sur $\mathbf{k}$ et par suite $\mathbf{k}_{2}^{(1)}$ sera égal à $\mathbf{k}(\sqrt{2}, \sqrt{p})$ et sera abélien sur $\mathbf{Q}$. D'où $\mathbf{k}_{2}^{(1)}=\mathbf{k}^{(*)}$, ce qui n'est pas le cas. Par conséquent, $\epsilon=i^{m} \epsilon_{1}$ pour un certain entier $m$. Il vient ensuite que $\sigma(\epsilon)= \pm i^{m} \sqrt{i \epsilon_{0}^{\prime}}$ où $\epsilon_{0}^{\prime}$ est le conjugué de $\epsilon_{0}$ et $\sigma(\epsilon)=\sigma(x)^{2}+4 \frac{\sigma(r)}{\sigma(s)}$, où $\sigma(r)$ et $\sigma(s)$ restent des entiers de $\mathbf{k}$ et $\sigma(s)$ reste premier avec 2. D'où $\mathbf{k}(\sqrt{\sigma(\epsilon)})$ est non-ramifié sur k. D'autre part, $\epsilon \sigma(\epsilon)=i^{m} \sqrt{i \epsilon_{0}} \times\left( \pm i^{m}\right) \sqrt{i \epsilon_{0}^{\prime}}= \pm i$ n'est pas un carré dans $\mathbf{k}$. Donc $\mathbf{k}(\sqrt{\epsilon}) \neq \mathbf{k}(\sqrt{\sigma(\epsilon)})$ et $\mathbf{k}(\sqrt{\epsilon \sigma(\epsilon)})$ est non-ramifié sur $\mathbf{k}$, ce qui n'est pas possible d'après le cas $\epsilon=i$. On en déduit que $\mathbf{k}$ n'est pas de type unité. Donc $\mathbf{k}$ est de type classe.

Théorème 10. Soit $\mathbf{k}=\mathbf{Q}(\sqrt{p q}, i)$ avec $p$ et $q$ deux nombres premiers tels que $q \equiv-1 \bmod 4, p \equiv 1 \bmod 8,\left(\frac{p}{q}\right)=-1$. Soit $\epsilon$ l'unité fondamentale de $\mathbf{Q}(\sqrt{p})$. Alors $\mathbf{k}_{2}^{(1)}=\mathbf{k}(\sqrt{p})(\sqrt{\epsilon})$. 
Preuve. Montrons que $\mathbf{k}_{2}^{(1)}=\mathbf{k}(\sqrt{p})(\sqrt{\epsilon})$. On sait d'après Cohn [9], que si $p \equiv 1 \bmod 8$, alors $\mathbf{k}_{1}=\mathbf{Q}(\sqrt{p}, i)(\sqrt{\epsilon})$ est une extension cyclique nonramifiée sur $\mathbf{Q}(\sqrt{-p})$. Soit $G_{0}$ le groupe de Galois de $\mathbf{k}_{1} / \mathbf{Q}$ et $\epsilon^{\prime}$ le conjugué de $\epsilon$, alors $G_{0}$ est engendré par les automorphismes $\sigma$ et $\tau_{1}$ définis par:

\begin{tabular}{|c||c|c|c|c|}
\hline & $\sqrt{p}$ & $i$ & $\sqrt{\epsilon}$ & $\sqrt{\epsilon^{\prime}}$ \\
\hline$\sigma$ & $-\sqrt{p}$ & $-i$ & $\sqrt{\epsilon^{\prime}}$ & $-\sqrt{\epsilon}$ \\
\hline$\tau_{1}$ & $-\sqrt{p}$ & $i$ & $\sqrt{\epsilon^{\prime}}$ & $\sqrt{\epsilon}$ \\
\hline
\end{tabular}

De plus on a que $\sigma^{4}=\tau_{1}^{2}=\left(\sigma \tau_{1}\right)^{2}=1$. Comme $\mathbf{L}=\mathbf{k}(\sqrt{p})(\sqrt{\epsilon})=$ $\mathbf{k}_{1} \mathbf{Q}(\sqrt{q})$; on peut prolonger $\sigma$ et $\tau_{1}$ par l'identité à $\mathbf{L}$. De même, si on désigne par $\tau_{2}$ l'automorphisme défini sur $\mathbf{Q}(\sqrt{q})$ par $\tau_{2}(\sqrt{q})=-\sqrt{q}$, alors on peut prolonger $\tau_{2}$ par l'identité à $\mathbf{L}$. Par suite, le groupe de Galois de $\mathbf{L} / \mathbf{Q}$ est engendré par $\sigma, \tau_{1}$ et $\tau_{2}$. On va déterminer le groupe de Galois de $\mathbf{L} / \mathbf{k}$. Il est clair que $\sigma^{2}$ et $\tau_{1} \tau_{2}$ laissent fixe $\mathbf{k}$. D'autre part $\sigma^{2}(\sqrt{p})=$ $\sqrt{p}, \tau_{1} \tau_{2}(\sqrt{p})=-\sqrt{p}, \sigma^{2}(\sqrt{\epsilon})=-\sqrt{\epsilon}, \tau_{1} \tau_{2}(\sqrt{\epsilon})=\sqrt{\epsilon^{\prime}},\left(\sigma^{2}\right)^{2}=\left(\tau_{1} \tau_{2}\right)^{2}=1$ et $\sigma^{2} \tau_{1} \tau_{2} \neq 1$. Donc le groupe engendré par $\sigma^{2}$ et $\tau_{1} \tau_{2}$ laisse fixe $\mathbf{k}$ et il est de type $(2,2)$. De plus, on a que $\mathbf{k}(\sqrt{p})$ est non-ramifié sur $\mathbf{k}$ et comme $\mathbf{k}_{1}$ est non-ramifié sur $\mathbf{Q}(\sqrt{p}, i)$, alors $\mathbf{L}$ est non-ramifié sur $\mathbf{Q}(\sqrt{q}) \mathbf{Q}(\sqrt{p}, i)=$ $\mathbf{k}(\sqrt{p})$. D'où $\mathbf{L}$ est non-ramifié sur $\mathbf{k}$ et le groupe de Galois de $\mathbf{L} / \mathbf{k}$ est de type $(2,2)$. Par conséquent $\mathbf{L}=\mathbf{k}_{2}^{(1)}$.

Théorème 11. Soit $\mathbf{k}=\mathbf{Q}(\sqrt{p q}, i)$ avec $p$ et $q$ deux nombres premiers tels que $q \equiv-1 \bmod 4, p \equiv 1 \bmod 8,\left(\frac{p}{q}\right)=-1$. Alors il existe deux entiers $a, b \in \mathbf{N}$ tels que $p=a^{2}+16 b^{2}$. Soient $\pi_{1}=a+4 b i, \pi_{2}=a-4 b i, \mathcal{H}_{1}$ et $\mathcal{H}_{2}$ les idéaux premiers au-dessus de $\pi_{1}$ et $\pi_{2}$ dans $\mathbf{k}$. Alors la 2-partie du groupe de classes de $\mathbf{k}$ est engendré par les classes de $\mathcal{H}_{1}$ et $\mathcal{H}_{2}$ et les trois extensions quadratiques non-ramifiées sur $\mathbf{k}$ sont: $\mathbf{k}^{(*)}=\mathbf{k}(\sqrt{p}), \mathbf{K}_{1}=$ $\mathbf{k}\left(\sqrt{\pi_{1}}\right)$ et $\mathbf{K}_{2}=\mathbf{k}\left(\sqrt{\pi_{2}}\right)$. De plus si $\mathbf{k}_{2}^{(2)} \neq \mathbf{k}_{2}^{(1)}$, alors seules la classe de $\mathcal{H}_{1}$ et son carré capitulent dans $\mathbf{K}_{1}$ et il en est de même pour $\mathbf{K}_{2}$, c'est-à-dire seules la classe de $\mathcal{H}_{2}$ et son carré capitulent dans $\mathbf{K}_{2}$.

Preuve. On sait, d'après Barruccand et Cohn [7], que si $p \equiv 1 \bmod 8$, alors il existe deux entiers a et b tels que $p=a^{2}+16 b^{2}$. Il est clair que $a$ est impair et donc $a \equiv \pm 1 \bmod 4$. On pose $\pi_{1}=a+4 b i$ et $\pi_{2}=a-4 b i$. Comme $-1=i^{2}$, alors $\pi_{1} \equiv x^{2} \bmod 4$ et $\pi_{2} \equiv x^{2} \bmod 4$ sont résolubles. Les nombres $\pi_{1}$ et $\pi_{2}$ sont des premiers ramifés dans $\mathbf{k} / \mathbf{Q}(i)$. Par suite, il existe deux idéaux de $\mathbf{k}, \mathcal{H}_{1}$ et $\mathcal{H}_{2}$ tels que $\mathcal{H}_{1}^{2}=\left(\pi_{1}\right)$ et $\mathcal{H}_{2}^{2}=\left(\pi_{2}\right)$. Par conséquent, $\mathbf{K}_{1} / \mathbf{k}$ et $\mathbf{K}_{2} / \mathbf{k}$ sont non-ramifiés. Comme $\mathbf{k}$ est de type classe, alors la 2-partie du groupe des classes de $\mathbf{k}$ est engendrée par les classes de $\mathcal{H}_{1}$ et $\mathcal{H}_{2}$. D'autre part, $\mathcal{H}_{1}^{2}=\left(\left(\sqrt{\pi_{1}}\right)^{2}\right)$ entraîne que l'idéal engendré par $\mathcal{H}_{1}$ dans $\mathbf{K}_{1}=\mathbf{k}\left(\sqrt{\pi_{1}}\right)$ est égal à l'idéal $\left(\sqrt{\pi_{1}}\right)$. Ceci veut dire que $\mathcal{H}_{1}$ capitule 
dans $\mathbf{k}_{1}$. Il en est de même pour $\mathcal{H}_{2}$ dans $\mathbf{k}_{2}$. Ainsi, si $\mathbf{k}_{2}^{(2)} \neq \mathbf{k}_{2}^{(1)}$, comme toutes les classes de $\mathbf{C}_{2}$ capitulent dans $\mathbf{k}^{(*)}$, alors seulement la classe de $\mathcal{H}_{1}$ et son carré capitulent dans $\mathbf{K}_{1}$ et seulement la classe de $\mathcal{H}_{2}$ et son carré capitulent dans $\mathbf{K}_{2}$.

Théorème 12. Soit $\mathbf{k}=\mathbf{Q}(\sqrt{p q}, i)$ avec $p$ et $q$ deux nombres premiers tels que $q \equiv-1 \bmod 4, p \equiv 1 \bmod 8,\left(\frac{p}{q}\right)=-1$. Soient $\mathbf{L}=\mathbf{Q}(\sqrt{-p})$ et $\mathbf{L}_{2}^{(1)}$ le 2-corps de classes de Hilbert de $\mathbf{L}$, alors $\mathbf{k}_{2}^{(2)}=\mathbf{k}^{(*)} \mathbf{L}_{2}^{(1)}$.

Preuve. D'après le théorème précédent, $\mathbf{K}_{1}$ et $\mathbf{K}_{2}$ sont conjugués. Par suite $\operatorname{Gal}\left(\mathbf{k}_{2}^{(2)} / \mathbf{K}_{2}\right)$ et $\operatorname{Gal}\left(\mathbf{k}_{2}^{(2)} / \mathbf{K}_{1}\right)$ sont conjugués et les 2-groupes de classes de $\mathbf{K}_{1}$ et $\mathbf{K}_{2}$ ont la même structure. D'autre part, d'après le Théorème 2 , si $\mathbf{k}_{2}^{(2)} \neq \mathbf{k}_{2}^{(1)}$, alors les 2-groupes de classes des corps $\mathbf{k}^{(*)}=\mathbf{k}(\sqrt{p}), \mathbf{K}_{1}=$ $\mathbf{k}\left(\sqrt{\pi_{1}}\right)$ et $\mathbf{K}_{2}=\mathbf{k}\left(\sqrt{\pi_{2}}\right)$ sont cycliques ou bien un seul corps parmi ces derniers corps est de 2-groupe de classes cyclique. Ainsi le 2-groupe de classes de $\mathbf{k}^{(*)}$ est cyclique. Par suite $\mathbf{k}_{2}^{(2)}=\left(\mathbf{k}^{(*)}\right)_{2}^{(1)}$. En calculant la 2partie du nombre de classes de $\mathbf{k}^{(*)}$ on voit que si $\mathbf{L}_{2}^{(1)}$ est le 2-corps de classes de Hilbert de $\mathbf{L}$, alors $\mathbf{k}_{2}^{(2)}=\mathbf{k}^{(*)} \mathbf{L}_{2}^{(1)}$.

Remarque 13. Toute l'étude faite dans ce paragraphe pour le cas $d=p q$ est aussi valable pour le cas $d=p_{1} p_{2}$ où $p_{1}$ et $p_{2}$ sont deux nombres premiers tels que $p_{1} \equiv 1 \bmod 8, p_{2} \equiv 5 \bmod 8$ et $\mathbf{C}_{\mathbf{k}, 2}$, le 2 -groupe des classes de $\mathbf{k}$, est de type $(2,2)$ (pour plus de détails pour ce cas voir [4]).

\section{References}

[1] A. Azizi, Sur la capitulation des 2-classes d'idéaux de $\mathbf{Q}(\sqrt{d}, i)$, C.R. Acad. Sci. Paris, 325, Série I, 1997, 127-130, MR 98d:11131, Zbl 0885.11061.

$[2]$, Sur le groupe de classes d'idéaux de $\mathbf{Q}(\sqrt{d}, i)$, Rendiconti del Circolo Matematico di Palermo, 47 (1998), 71-92.

[3] _ Unités de certains corps de nombres imaginaires et abéliens sur $\mathbf{Q}$, Annales des Sciences Mathématiques du Québec, 23(1) (1999), 15-21, MR 2000k:11120.

[4] Capitulation of the 2-ideal classes of $\mathbf{Q}\left(\sqrt{p_{1}}, \sqrt{-p_{2}}\right)$, Lecture Notes in Pure and Applied Mathematics, 208, 13-19, Marcel Dekker, New York, Basel, 2000, MR 2000h:11118.

[5] Capitulation des 2-classes d'idéaux de $\mathbf{Q}(\sqrt{2 p q}, i)$, Acta Arithmetica, XCIV.4 (2000), 383-399, MR 2001k:11221, Zbl 0953.11033.

[6] Sur une question de capitulation, Proc. Amer. Math. Soc., 130(8) (2002), 2197-2202 (electronic), CMP 1897477.

[7] P. Barruccand and H. Cohn, Note on primes of type $x^{2}+32 y^{2}$, class number, and residuacity, J. Reine Angew. Math., 238 (1969), 67-70, MR 40 \#2641, Zbl 0207.36202.

[8] S.M. Chang and R. Foote, Capitulation in class field extensions of type $(p, p)$, Can. J. Math., 32(5) (1980), 1229-1243, MR 82i:12013, Zbl 0459.12007. 
[9] H. Cohn, The explicit Hilbert 2-cyclic class fields of $\mathbf{Q}(\sqrt{-p})$, J. Reine Angew. Math., 321 (1981), 64-77, MR 82e:12011, Zbl 0455.12006.

[10] Introduction to the Construction of Class Fields, Cambridge University Press, 1985, MR 87i:11165, Zbl 0571.12001.

[11] F.P. Heider und B. Schmithals, Zur kapitulation der idealklassen in unverzweigten primzyklischen erweiterungen, J. Reine Angew. Math., 336 (1982), 1-25, MR 84g:12002, Zbl 0505.12016.

[12] H. Kisilevsky, Number fields with class number congruent to $4 \bmod 8$ and Hilbert's Theorem 94, J. Number Theory, 8 (1976), 271-279, MR 54 \#5188, Zbl 0334.12019.

[13] K. Miyake, Algebraic investigations of Hilbert's Theorem 94, the principal ideal theorem and capitulation problem, Expos. Math., 7 (1989), 289-346, MR 90k:11144, Zbl 0704.11048.

[14] H. Suzuki, A generalization of Hilbert's Theorem 94, Nagoya Math. J., 121 (1991), 161-169, MR 92h:11098, Zbl 0728.11061.

[15] F. Terada, A principal ideal theorem in the genus fields, Tôhoku Math. J. Second Series, 23(4) (1971), 697-718, MR 46 \#5285, Zbl 0243.12003.

Received July 30, 2001 and revised November 28, 2001.

DÉpartement de Mathématiques

FACUlté DES SCIENCES

Université Mohammed 1

OUJDA

MAROCCO

E-mail address: azizi@sciences.univ-oujda.ac.ma 